\title{
A Parametric Study of the Peel Test
}

\author{
M. D. Thouless ${ }^{*}$ and Q. D. Yang ${ }^{\S}$ \\ "Department of Mechanical Engineering \\ Department of Materials Science \& Engineering \\ University of Michigan \\ Ann Arbor, MI 48109-2125 \\ ${ }^{\S}$ Dept. Mechanical E Aerospace Engineering \\ University of Miami \\ Coral Gables, FL 33124-0624
}

\begin{abstract}
The force required to peel a film from a substrate is generally a complex function of geometry, the constitutive properties of the film and substrate, and the interfacial cohesive properties. In most analyses, the effects of the transverse shear force that is an integral aspect of almost any peel test are neglected, although they can be incorporated in an indirect fashion through models that invoke a root-rotation angle. In this study, a complete elastic solution that incorporates all the components contributing to crack-tip deformation, including bending moment, transverse shear force and axial force, is derived in a selfconsistent way. In particular, it is shown that, for a strong interface that requires a reasonably large peel strain, the transverse shear results in a significant deviation of the phase angle from earlier analyses that neglected the shear term. The present analysis also links the transverse shear component to the root-rotation angle. A cohesive-zone analysis is presented for the peeling of an elastic-plastic film. In this analysis the interface is modeled using cohesive elements, and the film is modeled by a full, two-dimensional, finite-element analysis. This analysis allows the full effects of bending, axial loading, and transverse shear to evolve, with no a-priori assumptions being made about their relative magnitudes. The numerical results show how the peel force depends on the film thickness. When the film is relatively thin, the peel force increases with an increase in thickness as the extent of plasticity increases. This increase in plasticity is associated with (i) an increase in the contribution of bending to the deformation at the crack tip, relative to the contribution of transverse shear, and (ii) an increase in the physical limits imposed by the dimensions of the film on the volume of any crack-tip plastic zone. When the film is relatively thick, elasticity dominates the deformation of the film, and small-scale yielding effects become important. The peel force is dictated by the toughness of the interface and by crack-tip plasticity (if any) induced by the cohesive stresses. Therefore, peel forces tend to minimum values for both thick and thin films. A maximum peel force is exhibited for films with an intermediate thickness.
\end{abstract}

Keywords: Peel test, fracture, elasto-plasticity

(July 18, 2008) 


\section{Nomenclature}

E Young's modulus of film

$\bar{E} \quad E /\left(1-v^{2}\right)$ in plane strain, and $E$ in plane stress

$E_{s} \quad$ Young's modulus of substrate

$G \quad$ Energy-release rate

G Mode-I component of energy-release rate

$G_{\text {II }} \quad$ Mode-II component of energy-release rate

h $\quad$ Film thickness

$M \quad$ Bending moment (per unit width) acting at crack tip

$n \quad$ Power-law hardening exponent for film

$N \quad$ Axial (compressive) force (per unit width) acting at crack tip

$P \quad$ Force (per unit width) applied to film

$P_{f} \quad$ Peel force (per unit width) required to cause delamination

$V \quad$ Transverse shear force (per unit width) acting at crack tip

$\alpha \quad$ Primary dimensionless modulus mismatch ratio

$\beta \quad$ Secondary dimensionless modulus mismatch ratio

$\Gamma \quad$ Interfacial toughness

$\Gamma_{\mathrm{I}} \quad$ Mode-I component of interfacial toughness (area under mode-I tractionseparation law)

$\Gamma_{\text {II }} \quad$ Mode-II component of interfacial toughness (area under mode-II tractionseparation law)

$\theta \quad$ Peel angle

$v \quad$ Poisson's ratio of film

$v_{\mathrm{s}} \quad$ Poisson's ratio of substrate

$\hat{\sigma} \quad$ Mode-I cohesive strength of interface

$\sigma_{Y} \quad$ Yield strength of film

$\hat{\tau} \quad$ Mode-II cohesive strength of interface

$\psi \quad$ Phase angle 


\section{Introduction}

Owing to its simplicity of concept and geometry, the peel test is popular for adhesion measurements. The geometry consists of a film bonded to a thick substrate, and the test proceeds by measuring the force required to pull the film off the substrate. This peel force is then related to the properties of the interface. Under some limiting conditions, the peel force is a direct measure of the interfacial toughness. However, more generally, the peel force is affected by the geometry, the constitutive properties of the film and substrate, and the cohesive properties of the interface. The geometrical terms include the peel angle, $\theta$, and the film thickness, $h$, (Fig. 1). If the film and substrate are both isotropic and elastic, then the relevant constitutive properties are the Young's moduli, E and $E_{s^{\prime}}$ and Poisson's ratio, $v$ and $v_{s^{\prime}}$ of the film and substrate. The yield strength and hardening characteristics of the film enter the problem if there is plasticity. For the purposes of this paper, it was assumed that the substrate is very hard, so that yield did not occur at any scale within the substrate. The film was assumed to have a yield strength of $\sigma_{Y}$, with a power-law hardening relationship after yield, so that the true strain, $\widetilde{\varepsilon}$, and true stress, $\widetilde{\sigma}$ were related by:

$$
\widetilde{\varepsilon}=\frac{\sigma_{Y}}{\bar{E}}\left(\frac{\widetilde{\sigma}}{\sigma_{Y}}\right)^{1 / n} \quad \text { for } \widetilde{\sigma} \geq \sigma_{Y},
$$

where $n$ is the power-law hardening exponent, and $\bar{E}=E$ in plane stress, and $\bar{E}=E /\left(1-v^{2}\right)$ in plane strain.

The cohesive properties of the interface were assumed to be described by mode-I and mode-II traction-separation laws, and a mixed-mode failure criterion that couples them. In general, the important features of traction-separation laws 
are the mode-I toughness, $\Gamma_{\mathrm{I}}$, the normal cohesive strength, $\hat{\sigma}$, the mode-II toughness, $\Gamma_{\mathrm{II}}$ and the shear cohesive strength, $\hat{\tau} .{ }^{1}$ Other details of the laws, such as the shape, generally seem to have a minor role on the fracture process: they affect details of the fracture, but not the fundamental conclusions that will be emphasized in this paper. For the purposes of this paper, a simple shape for the traction-separation laws was used, as illustrated in Fig. 2. These two laws were linked with a simple mixed-mode fracture criterion [Yang and Thouless, 2001]

$$
\frac{G_{I}}{\Gamma_{I}}+\frac{G_{I I}}{\Gamma_{I I}}=1
$$

where $G_{\mathrm{I}}$ and $G_{\mathrm{II}}$ are the mode-I and mode-II components of the energy-release rate, such that the total energy release rate is given by

$$
G=G_{\mathrm{I}}+G_{\mathrm{II}}
$$

The mode-I and mode-II components of the energy-release rate are defined by

$$
G_{I}=\int_{0}^{\delta_{n}} \sigma d \delta_{n} \text { and } G_{I I}=\int_{0}^{\delta_{t}} \tau d \delta_{t} .
$$

The mode-I and mode-II toughness are defined by

$$
\Gamma_{I}=\int_{0}^{\delta_{n c}} \sigma d \delta_{n} \text { and } \Gamma_{I I}=\int_{0}^{\delta_{t c}} \tau d \delta_{t}
$$

where the mode-I traction is $\sigma$, the mode-I separation is $\delta_{n}$, the mode-II traction is $\tau$, the mode-II separation is $\delta_{t}$, and the critical displacements at which the tractions go to zero in each mode are $\delta_{n c}$ and $\delta_{t c}$.

\footnotetext{
${ }^{1}$ Other parameters that can be used as possible characterizations of the cohesive properties of the interface include a critical root rotation, critical displacement or critical strain. These are essentially variations on a theme, and can be re-expressed in terms of the cohesive strength and toughness.
} 
The relative ratio between the two modes of energy-release rate is described by a phase angle, defined as [Yang and Thouless, 2001; Parmigiani and Thouless, 2007]:

$$
\psi=\tan ^{-1} \sqrt{G_{I I} / G_{I}} .
$$

Linear-elastic fracture mechanics is predicated on the use of the energy-based failure criterion, $G=\Gamma$, where $\Gamma$ is the toughness at the appropriate phase angle. Equations 2, 3 and 6 can be re-expressed in terms of a mixed-mode failure criterion of

$$
\frac{\Gamma}{\Gamma_{I}}=\Gamma(\psi)=\frac{\lambda\left(1+\tan ^{2} \psi\right)}{\lambda+\tan ^{2} \psi},
$$

where $\lambda=\Gamma_{\mathrm{II}} / \Gamma_{\mathrm{I}}$, which follows the general form of mixed-mode failure criteria often used in the fracture-mechanics literature. The numerical analyses presented in this paper have been conducted with $\lambda=1$. This is modeindependent fracture, for which mixed-mode effects do not play a role.

\section{Parametric description of the peel geometry}

In general, the peel force, $P_{f}$, depends on all the geometrical and material parameters, so that

$$
\frac{P_{f}}{\Gamma_{I}}=f\left(\frac{\bar{E} h}{\Gamma_{I}}, \frac{\Gamma_{I I}}{\Gamma_{I}}, \theta, \frac{\sigma_{Y}}{\bar{E}}, n, \frac{\hat{\sigma}}{\sigma_{Y}}, \frac{\hat{\tau}}{\sigma_{Y}}, \frac{\bar{E}_{s}}{\bar{E}}, v, v_{s}\right) .
$$

There are, however, limiting regimes under which some of these dimensionless groups can be neglected. 


\subsection{Completely-elastic limit}

The simplest regime is one in which energy dissipation occurs only in the interface, with no plasticity in the film. The peel test can then be analyzed in terms of the energy-based failure criterion associated with linear-elastic fracture mechanics. Under these conditions, a steady-state energy balance can be used to derive a simple expression for the peel force in terms of the mode-dependent toughness of the interface. Assuming that strains are not so extensive so as to preclude the use of nominal stresses and strains, ${ }^{2}$ the energy-release rate, $G$, associated with an applied force, $P$, is given by Kendall [1975]:

$$
\frac{G}{\bar{E} h}=\frac{P}{\bar{E} h}(1-\cos \theta)+0.5\left(\frac{P}{\bar{E} h}\right)^{2} \text {. }
$$

Fracture occurs when the energy-release rate is equal to the mode-dependent toughness of the interface. Therefore, the peel force is given by

$$
\frac{P_{f}}{\Gamma_{I}}=-\frac{\bar{E} h}{\Gamma_{I}}(1-\cos \theta)+\sqrt{\left(\frac{\bar{E} h}{\Gamma_{I}}\right)^{2}(1-\cos \theta)^{2}+\frac{2}{\Gamma(\psi)} \frac{\bar{E} h}{\Gamma_{I}}} .
$$

For mode-independent failure, when $\Gamma(\psi)=1$, Eqn. 10 gives an expression for the peel force that is a simple function of $\Gamma_{I} / \bar{E} h$ and $\theta$ only. However, the phase angle must also be calculated to evaluate the peel force for modedependent failure. In general, the phase angle depends on the peel force, the elastic properties of the film and substrate, and the cohesive properties of the interface [Parmigiani and Thouless, 2007]. Therefore, for mode-dependent failure, the peel force for an elastic film is of the form

\footnotetext{
${ }^{2}$ For large strains, with conservation of volume and plane stress, this expression becomes $G / E h=\ln \left(1+\varepsilon_{n}\right)\left[(1-\cos \theta)-0.5 \ln \left(\left(1+\varepsilon_{n}\right)\right]\right.$, where $\varepsilon_{n}$ is defined by $P / E h=\ln \left(1+\varepsilon_{n}\right) /\left(1+\varepsilon_{n}\right)$. A limit on the peel force is set by $\varepsilon_{\mathrm{n}}=1.73$.
} 


$$
\frac{P_{f}}{\Gamma_{I}}=f\left(\frac{\bar{E} h}{\Gamma_{I}}, \frac{\Gamma_{I I}}{\Gamma_{I}}, \frac{\hat{\sigma}}{\bar{E}}, \frac{\hat{\boldsymbol{\tau}}}{\hat{\sigma}}, \theta, \alpha, \beta\right) .
$$

In this expression, plane conditions have been assumed so that the two dimensionless groups

$$
\alpha=\frac{\bar{E}-\bar{E}_{s}}{\bar{E}+\bar{E}_{s}}
$$

and

$$
\beta=\frac{\bar{E} g\left(v_{s}\right)-\bar{E}_{s} g(v)}{\bar{E}+\bar{E}_{s}}
$$

with $g(v)=(1-2 v) /[2(1-v)]$ in plane strain, and $g(v)=(1-2 v) / 2$ in plane stress, replace the three dimensionless groups that are generally required to describe isotropic modulus mismatch [Dundurs, 1969]. If the fracture-length scales, $\bar{E} \Gamma_{I} / \hat{\sigma}^{2} h$ and $\bar{E} \Gamma_{I I} / \hat{\tau}^{2} h$ are sufficiently small for linear-elastic conditions to apply, the cohesive strengths of the interface do not affect the phase angle, and the peel force is dependent on five dimensionless groups:

$$
\frac{P_{f}}{\Gamma_{I}}=f\left(\frac{\Gamma_{I I}}{\Gamma_{I}}, \frac{\bar{E} h}{\Gamma_{I}}, \theta, \alpha, \beta, .\right.
$$

Thouless and Jensen [1992] presented a derivation for the phase angle, from which the peel force can be calculated for a given mixed-mode failure criterion. However, this derivation neglected the effect of shear at the crack tip. Therefore, a corrected derivation for the phase angle is given in Section 3 of this paper.

\subsection{Plasticity}

There are two distinct sources of plasticity in the peel geometry: (i) cracktip plasticity induced by the cohesive tractions [Wei and Hutchinson, 1998], and (ii) bending plasticity [Kim and Aravas, 1988; Kim et al., 1989]. If $\hat{\sigma} / \sigma_{Y}$ is greater 
than about two, local yielding at the crack tip occurs in response to the cohesive tractions [Wei and Hutchinson, 1998]. The peel force is then elevated above the level predicted by Eqn. 10, as a direct consequence of the additional energy dissipated by this crack-tip plasticity induced by the fracture process. If the effects of bending plasticity are neglected, the size of the crack-tip plastic zone is limited by the film thickness when the film is relatively thin. This results in an approximately linear dependence between the peel force and the film thickness, until the film is thick enough for a fully-developed plastic zone to evolve. When the film is relatively thick, the crack-tip plasticity is embedded in a linear elastic region. In this small-scale yielding limit, the peel force is determined by the equations of linear-elasticity given in the previous section, with the toughness being replaced by a small-scale yielding toughness, $\Gamma_{\text {ssy }}$ that incorporates the energy dissipated by crack-tip plasticity in addition to the energy dissipated at the interface. This small-scale yielding value of toughness depends on the cohesive properties of the interface, as well as on the constitutive properties of the film and substrate. Therefore, the peel force is a function of all the parameters listed in Eqn. 8, as shown in Wei and Hutchinson [1998] and Tvergaard and Hutchinson [1992; 1996].

When the non-dimensional group $\bar{E} \Gamma_{I} / \sigma_{Y}^{2} h$ is greater than about $1 / 6$, the bending moment acting at the crack tip can induce macroscopic plasticity [Kim and Aravas, 1988; Kim et al. 1989]. This bending plasticity can occur even when the cohesive strengths are too low to trigger any crack-tip plasticity. A cohesivezone analysis by Yang et al. [2000] of this regime was limited to a range of parameters for which elastic deformations could be neglected. In general, all 
seven dimensionless groups of Eqn. 8 are required to describe the peel force, because the interfacial cohesive strengths induce a degree of triaxiality in the film, and affect the distribution of bending stresses. The analysis of Yang et al. [2000] indicated that the peel force increased with film thickness, but less strongly than the linear dependence shown by Wei and Hutchinson [1998] that was associated with fracture-induced, crack-tip plasticity. A subsequent paper by Wei [2004], confirmed this weaker dependence on the peel force when plasticity was associated only with bending.

\section{Phase angle for the elastic peel test}

The peel force can be determined from a steady-state energy balance, provided the toughness of the interface is mode-independent. If the toughness varies with phase angle, knowledge of the phase angle is required to analyze the peel test. The peel geometry belongs to a class of fracture problems that involves the delamination of beams. General solutions for the energy-release rate and phase angle have been developed in terms of the axial force, bending moment, and shear forces acting at the crack tip. For the peel geometry, attention can be limited to the solutions appropriate for infinitely-thick substrates. For a beam with an axial compression, $N$ (per unit width), applied at the crack tip (Fig. 3a), the energy-release rate is given by

$$
G_{N}=0.5 \frac{N^{2}}{\bar{E} h}
$$

with a phase angle $\psi_{\mathrm{N}}=\psi_{\mathrm{N}}(\alpha, \beta)$ which is given in Suo \& Hutchinson [1990]. When, $\alpha=\beta=0, \psi_{N}=+52.1^{\circ}$ (where a positive sign indicates shear in a sense that would cause the crack to kink into the substrate). For a beam with a bending 
moment, $M$ (per unit width) applied at the crack tip (Fig. 3b), the energy-release rate is given by

$$
G_{M}=6 \frac{M^{2}}{\bar{E} h^{3}}
$$

with a phase angle $\psi_{\mathrm{M}}(\alpha, \beta)=-90^{\circ}+\psi_{\mathrm{N}}(\alpha, \beta)$. For a beam with a transverse shear force, $V$ (per unit width) applied at the crack tip (Fig. 3c), the energy-release rate is given by:

$$
G_{V}=f_{v}^{2}(\alpha, \beta) \frac{V^{2}}{\bar{E} h}
$$

with a phase angle $\psi_{\mathrm{V}}=\psi_{\mathrm{V}}(\alpha, \beta) . f_{V}(\alpha, \beta)$ is a function of $\alpha$ and $\beta$ and can be found along with the phase angle in Li et al. [2004] (for $\beta=0$ ). For the case of $\alpha=\beta=0$,

$$
G_{V}=3.764 \frac{V^{2}}{\bar{E} h}
$$

and $\psi_{\mathrm{V}}=+0.7^{\circ}$. If the three loading components act together in a single geometry, the energy-release rate and the phase angle are given by [Li et al., 2004]

$$
\begin{aligned}
\bar{E} G & =\frac{N^{2}}{h}\left[0.5+\sqrt{2} f_{V}(\alpha, \beta)\left(\frac{V}{N}\right) \cos \left(\psi_{N}-\psi_{V}\right)\right] \\
& +\frac{M^{2}}{h^{3}}\left[6+f_{V}^{2}(\alpha, \beta)\left(\frac{V h}{M}\right)^{2}+2 \sqrt{6} f_{V}(\alpha, \beta)\left(\frac{V h}{M}\right) \cos \left(\psi_{V}-\psi_{M}\right)\right]
\end{aligned}
$$

and

$$
\psi=\tan ^{-1}\left\{\frac{\{N h / M\} \sin \psi_{N}+\sqrt{12} \sin \psi_{M}+\sqrt{2} f_{V}(\alpha, \beta)\{V h / M\} \sin \psi_{V}}{\{N h / M\} \cos \psi_{N}+\sqrt{12} \cos \psi_{M}+\sqrt{2} f_{V}(\alpha, \beta)\{V h / M\} \cos \psi_{V}}\right\}
$$

For a peel geometry, the energy-release rate is known from the steadystate energy balance, but the phase angle can not be computed without 
knowledge of the three loading components. If the axial force, moment and transverse force acting at the crack tip are known, then the phase angle can be computed. For a peel test, the axial force and transverse shear force can be approximated from equilibrium considerations as (Fig. 4):

$$
\begin{aligned}
& N=-P \cos \theta \\
& V=P \sin \theta
\end{aligned}
$$

The bending moment at the crack tip is given by the product of the applied load times the distance from the crack tip to the line of action of the load (Fig. 4). This could be computed by elasticity calculations for a bending beam that represents the peeling film. Such calculations rely on appropriate assumptions about the boundary conditions at the point where the delaminated film joins the part of the film still attached to the substrate. The simplest assumption is that the film is rigidly clamped orthogonal to the substrate at the crack tip. However, this assumption neglects the effects of deformation in the film and substrate. Therefore, an alternative approach is to assume that the delaminated portion of the film is clamped at the crack tip, but rotated by an angle from the orthogonal orientation. This "root-rotation angle" depends on the axial load and bending moment acting at the crack tip [Cottrell and Chen (2000); Yu and Hutchinson (2002)]. It also depends on the shear force acting at the crack tip [Li et al., 2004; Andrews and Massabò, 2007]. An approximate expression for the average rotation (in radians) of the planes at the crack tip for a peel geometry given by $\mathrm{Li}$ et al. [2004] is

$$
\theta_{o}=c_{N} \frac{N}{\bar{E} h}+c_{M} \frac{M}{\bar{E} h^{2}}+\left(c_{V}-v\right) \frac{V}{\bar{E} h},
$$


where $c_{N}, c_{M}$, and $c_{V}$ are functions of the modulus mismatch between the film and substrate. For $\alpha=\beta=0$, the three numerical constants are given by $c_{N}=1.9$, $c_{M}=7.0$, and $c_{V}=3.1$. If this angle is known, the moment acting at the crack tip can be calculated by an analysis of the delaminated portion of the film, subjected to this rotated and clamped boundary condition.

An alternative approach to calculating the moment and the phase angle is to recognize that, once $N$ and $V$ have been calculated from equilibrium considerations (Eqn. 15), the bending moment can be found from a comparison between the two forms of the energy-release rate given by Eqns. (16a) and Eqn. (9). Provided the shear term is specifically included in the analysis, no assumptions are required about the nature of the crack-tip deformation, since the deformation is correctly incorporated in the expressions given above for the energy-release rates and phase angles.

As an example of the procedure outlined above, the special case of $\alpha=\beta=0$, with a peel angle of $\theta=90^{\circ}$ is considered. Substitution of Eqn. (17) into Eqn. (16a) results in an expression for the energy-release rate in terms of the nondimensional crack-tip bending moment, $\mathrm{M} / \mathrm{Ph}$ :

$$
\frac{\bar{E} G h}{P^{2}}=6.00\left(\frac{M}{P h}\right)^{2}+7.43\left(\frac{M}{P h}\right)+3.76 .
$$

A comparison of this expression with Eqn. 9, gives the result that

$$
\frac{M}{P h}=-0.619+0.408 \sqrt{\frac{\bar{E} h}{P}-0.965} .
$$


More generally, Fig. 5 shows how the crack-tip bending moment for a $90^{\circ}$-peel test varies with peel strain, for different values of modulus mismatch between the film and substrate. Superimposed on this plot is the crack-tip bending moment that was computed by the same process, but with the shear term being neglected [Thouless and Jensen, 1992]:

$$
\frac{M}{P h}=0.408 \sqrt{\frac{\bar{E} h}{P}+0.5}
$$

It can be seen from Fig. 5 that this earlier analysis is valid in the limit of very small peel strains, $P / \bar{E} h$, for which the root rotations are negligible. Indeed, Eqn. 20 is identical to the results of calculations based on clamped boundary conditions at the crack tip (root-rotation angle equals zero).

The observation that the effects of shear are significant for stiff films and high peel strains, can be used to make a connection to the body of literature that views the peel test through the prism of root rotation [Williams, 1993; Kinloch et al., 1994]. Root rotation effects are only important if shear effects are important and, at some level, the two effects should be regarded as manifestations of the same phenomenon [Li et al., 2004; Andrews and Massabò, 2006]. Root rotation becomes more pronounced as the peel strain increases (the loading terms of Eqn. 18), and as the compliance of the substrate increases (the constant terms of Eqn. 18). Root rotation occurs in the absence of shear, but steady-state arguments can be used to show that it has no effect on the delamination of a linear-elastic system loaded by a pure moment or by a pure axial load. ${ }^{3}$ Root

\footnotetext{
${ }^{3}$ Additional root rotation, beyond that identified by Eqn. 18, can also be associated with deformation of the interface region, such as an adhesive layer. Parmigiani and Thouless [2007] have shown that the linear-elastic results for films, such as those used in this text, are very robust
} 
rotation only has an influence on fracture through its interaction with the shear component of loading [Andrews and Massabò, 2007]. Therefore, if the effects of shear are neglected (as in Thouless and Jensen [1992]), the analysis reduces to one for which root rotation is ignored, as would be appropriate for small peel strains and rigid substrates. To further make the connection between the present analysis, and the root-rotation perspective, a plot of root-rotation angle against peel strain for different values of $\alpha$ is shown in Fig. 6. The effect of substrate compliance and peel strain on the root-rotation angle is apparent from this plot.

The phase angle can be determined by substituting the result for $V, N$ and $M$, into Eqn. 16b. It is plotted for $\alpha=\beta=0$ as a function of peel angle for different peel strains in Fig. 7. A comparison of this figure with the equivalent figure given in Thouless and Jensen [1992] reveals the effects of shear. If shear is neglected, the phase angle is constant at $-37.9^{\circ}$ for all values of peel strain in the $90^{\circ}$-peel test. However, when shear (and, consequently, root rotation) is included in the analysis, the mode-I component increases with peel strain for a peel angle of $90^{\circ}$, but the phase angles appears to be independent of peel strain at a peel angle of about $30.3^{\circ}$ (for $\alpha=\beta=0$ ). In contrast to the earlier results, these new calculations indicate that the magnitude of the phase angle decreases as the peel angle increases beyond $90^{\circ}$. The full solution indicates that the role of the mode-I component of the energy-release rate is a maximum for peel angles of about $90^{\circ}$, and the $90^{\circ}$-peel test is quite strongly mode-I for large peel strains.

even for relatively large fracture length scales defined by $E \Gamma_{I} / \hat{\sigma}^{2} h$ and $E \Gamma_{I I} / \hat{\tau}^{2} h$. Furthermore, even when they occur, fracture-length scales affect only phase angles and energy-release rates associated with shear. 
The calculation of the bending moment provided in this section can also be used to determine the conditions required to avoid plastic deformation of the film. Following the insight of Kim and co-workers [Kim and Aravas,1988; Kim et al., 1989] that yield is induced by the bending stresses at the crack tip, the requirement to avoid yield is given by

$$
\frac{\sigma_{Y}}{\bar{E}}>\frac{P}{\bar{E} h}\left[|\cos \theta|+6 \frac{M}{P h}\right],
$$

provided the cohesive stresses are sufficiently low that they do not affect the bending stresses. Furthermore, if the cohesive stresses do not induce plasticity, this equation can be re-expressed in terms of material parameters through Eqn. 10 and Eqn. 20. A plot of the resulting conditions to avoid yield is given in Fig. 8. Superimposed on this plot is the condition given by Kim and Aravas [1988] of $\sigma_{Y} / \bar{E}=\sqrt{6 \Gamma_{I} / \bar{E} h}{ }^{4}$ It will be observed that this provides an excellent description of the yield condition, when $\bar{E} h / \Gamma_{I}$ is greater than about 500 .

\section{Elastic-plastic analysis of the peel test}

In this section, the results of a cohesive-zone analysis for an elastic-plastic peel test are presented. The film was modeled using 2-D plane-strain continuum elements. Large-strain, large-rotation formulations were used for all numerical calculations. Plasticity in the film was modeled by a von Mises yield criterion (J-flow theory) coupled with isotropic hardening, and a uniaxial yield strength of $\sigma_{\mathrm{Y}}$. Power-law hardening occurred after yield, following Eqn. (1). This full numerical elastic-plastic modeling of the deformation of the film, which includes

\footnotetext{
${ }^{4}$ This equation can be derived by considering only the moment acting at the crack tip. From Eqn. 14, fracture occurs if $M=\sqrt{\bar{E} h^{3} \Gamma_{I} / 6}$. Yield occurs if $M>\sigma_{Y} h^{2} / 6$.
} 
all the effects of shear and axial loading, as well as bending, makes these analyses of the peel test distinct from earlier cohesive-zone analyses. The analysis followed the implementation of the mixed-mode cohesive-zone model described in Yang and Thouless [2001]. The interface was modeled using user-defined elements with the cohesive law shown in Fig. $2^{5}$ and the mixed-mode failure criterion of Eqn. 2. Boundary conditions appropriate for a rigid substrate were enforced. The numerical analyses were done by allowing delamination of the peel geometry to proceed until a steady-state peeling configuration was reached. The peel force under these steady-state conditions, $P_{f}$, was determined for different values of the normalized thickness, $\sigma_{Y} h / \Gamma_{I}$. All calculations were done for the following set of non-dimensional parameters: $n=0.2 ; \theta=90^{\circ} ; \hat{\boldsymbol{\tau}} / \sigma_{Y}=1$; $\sigma_{Y} / \bar{E}=0.00130$; and $\Gamma_{\mathrm{II}} / \Gamma_{\mathrm{I}}=1$. Two distinct conditions for the normal cohesive strength were examined. One set of calculations was done with a cohesive strength so low $\left(\hat{\sigma} / \sigma_{Y}=1\right)$, that crack-tip plasticity was not induced by the fracture process. The other set of calculations was done with $\hat{\sigma} / \sigma_{Y}=3$, so that the cohesive tractions induced crack-tip plasticity.

The normalized steady-state peel force, $P_{f} / \Gamma_{\mathrm{I}}$, is plotted as a function of the normalized film thickness, $\sigma_{\mathrm{Y}} h / \Gamma_{\mathrm{I}}$ in Fig. 9. The form of this plot follows the schematic form proposed by Gent and Ahmed [1977], with a peak peel force at intermediate values of film thickness. As the film increases in thickness, the volume of the plastically-deforming material (and, hence, the energy dissipated) increases. However, eventually, elastic bending begins to dominate over plastic

\footnotetext{
${ }^{5}$ Although many different cohesive laws can be proposed for use in such models, exploration of different laws indicated that the shape of the law does not have a major influence on the general features of fracture of interest in this study.
} 
bending. This reduces the energy dissipation and the peel force. These competing effects lead to the intermediate regime in which the peel force is a maximum.

In the limit of very large values of $\sigma_{\mathrm{Y}} h / \Gamma_{\mathrm{I}}$ no bending plasticity is associated with the deformation induced by the peel force. The only sources of energy dissipation are the toughness of the interface itself and any crack-tip plasticity triggered by the cohesive tractions. In particular, if the cohesive strength is so low that crack-tip plasticity does not occur, the peel force is given by Eqn. 10. For example, with a mode-independent, $90^{\circ}$-peel test the asymptotic peel force in this limit would be equal to $P_{f} / \Gamma_{\mathrm{I}}=1$. For the results shown in Fig. 9, this is the limit anticipated for the curve shown for $\hat{\sigma} / \sigma_{Y}=1$. Conversely, if the cohesive strength is large enough to trigger crack-tip plasticity, then for a $90^{\circ}$-peel test the asymptotic peel force is given by $P_{f}=\Gamma_{\text {ssy. }}$. A separate set of calculations was conducted to estimate the small-scale yielding toughness appropriate for $\hat{\sigma} / \sigma_{Y}=3$ and the other non-dimensional parameters used to obtain the results of Fig. 9. This calculation was conducted for a very thick film $\left(\sigma_{Y} h / \Gamma_{I}=4761\right)$, but for a simple cantilever-beam geometry where large rotations did not need to be considered. A point load was applied perpendicular to the interface at a distance $a$ from the crack tip. The load, $F$, required to propagate the crack was measured, and the toughness was computed from the LEFM expression

$$
\Gamma=6 F^{2} a^{2} / \bar{E} h^{3} .
$$

As expected, $R$-curve behavior was exhibited, with the toughness increasing as the crack grew. A steady-state value of about $\Gamma \approx 1.5 \Gamma_{\mathrm{I}}$ was obtained after the 
crack had grown by about 5\% of $h$. Based on this result, it is anticipated that the peel force shown for $\hat{\sigma} / \sigma_{Y}=3$ should reach an asymptotic limit of about $P_{f} / \Gamma_{I} \approx 1.5$ when $\sigma_{\mathrm{Y}} h / \Gamma_{\mathrm{I}}$ is large, as indicated in Fig. 9. However, the numerical difficulties associated with doing the full analysis of the peel test precluded examining the peel geometry in this regime. Nevertheless, it will be noted from Fig. 9 that, even for $\hat{\sigma} / \sigma_{Y}=3$, the calculations were conducted into a regime just past the peak load.

Towards the other limit, with low values of $\sigma_{\mathrm{Y}} h / \Gamma_{\mathrm{I}}$ energy is dissipated by bending-induced plasticity for both sets of parameters. A steady-state energy balance that ignored shear effects (with clamped boundary conditions at the crack tip), and only included the energy dissipated by plasticity in bending showed that the peel force should be independent of film thickness [Yang and Thouless, 2001]. For a mode-independent interface with small cohesive stresses and neglecting any elastic deformation, the peel force is given by this analysis as

$$
\frac{P_{f}}{\Gamma_{I}}=1+\frac{2^{n+1}}{n} .
$$

For a hardening exponent of $n=0.2$, this would correspond to a peel force of $12.5 \Gamma_{\mathrm{I}}$. This analysis that was predicated on three assumptions: (i) neglect of the shear contribution, (ii) neglect of the elastic contribution, and (iii) neglecting the cohesive-zone-induced plasticity.

It was argued in Yang and Thouless [2001] that as the film becomes thinner, the loading line moves towards the crack tip, so that transverse shear becomes significant relative to bending at the crack tip. This effect was observed 
in the numerical calculations done in this study, and reduces the extent of bending plasticity. The reduced level of bending plasticity results in the peel force dropping towards $\Gamma_{\mathrm{I}}$. As was shown in Section 3, shear effects also become important as the film becomes thinner under elastic conditions. When the cohesive stresses are large, there are the additional effects of crack-tip plasticity, as discussed by Wei and Hutchinson [1998] and Wei [2004]. The full elastoplastic numerical analysis presented in this paper that does not rely on an assumption of pure bending, and it allows all of these effects to be exhibited in the results presented in Fig. 9.

Figure 10 shows the deformation of the film with $\hat{\sigma} / \sigma_{Y}=3$ and $\sigma_{Y} h / \Gamma_{I}=4.76$ just before the crack propagation. Significant necking caused by local plastic flow at the crack-tip region can be seen. The von Mises stress distribution is not symmetrical about the (local) neutral axis, indicating a strong influence of the shear stress. This is further confirmed by the local grid distortion in the near-tip region shown in Figure 10(b). The maximum logarithmic shear strain in the immediate crack wake reaches about 200\%! Alternatively, the distortion of the mesh illustrates how this deformation can also be viewed as a root-rotation effect, although the rotation is not uniform through the thickness of the film.

\section{Conclusions}

Existing peel analyses generally neglect the transverse shear force that acts at the tip of the interface crack in peel geometries, although some of the effects are implicitly introduced through the use of a root-rotation description of the 
crack-tip deformation. In this paper, a complete elastic solution has been derived for the peel geometry that incorporates the full effects of crack-tip deformation and root rotation in a self-consistent manner, through the inclusion of the transverse shear force. The results of this analysis shows that if a strong interface requires a reasonably large peel strain, but still remains elastic, there is a significant error in the phase angle associated with neglecting the transverse shear component of the crack-tip loads.

The effects of transverse shear on elastic-plastic peel joints were investigated numerically, with the interface being simulated using cohesive elements. This numerical analyses included all the effects of transverse shear, axial loading, and bending, in conjunction with a full elastic-plastic constitutive law for the film. The numerical analyses showed that if the interface strength is too small to induce crack-tip plasticity, the normalized peel force $\left(P_{f} / \Gamma_{\mathrm{I}}\right)$ approaches unity for both very thin and very thick films, with a maximum peel force occurring at an intermediate thickness. The limit for thin films is associated with transverse shear, rather than bending, dominating the crack tip deformation. The limit for thick films is associated with the bending being entirely elastic. When the interface strength is sufficiently large for crack-tip plasticity to be induced, the behavior is similar, except the peel forces are increased owing to the additional effects of crack-tip plasticity. 


\section{References}

Andrews, MG. and R. Massabò, "The Effects of Shear and Near Tip Deformations on Energy Release Rate and Mode Mixity of Edge-Cracked Orthotropic Layers," Engineering Fracture Mechanics, in press (2007).

Cotterell, B and Z. Chen, "Buckling and Cracking of Thin Films on Compliant Substrate under Compression," International Journal of Fracture, 104, 169-179 (2000).

Dundurs, J., "Edge-bonded dissimilar orthogonal elastic wedges," Journal of Applied Mechanics, 32, 400-402 (1969)

Gent A. N. and G. R. Hamed, "Peel mechanics of adhesive joint", Polymer Engineering and Science, 17, 462-466 (1977).

Kendall, K., "Thin-film Peeling - the Elastic Term," Journal of Applied Physics, 8, 1449-1452 (1975).

Kim, K.-S. and N. Aravas, "Elasto-plastic Analysis of the Peel Test," International Journal of Solids and Structures, 24, 417-435 (1988).

Kim J., K. S. Kim and Y. H. Kim, "Mechanical effects in peel adhesion test", Journal of Adhesion Science and Technology, 3, 175-187 (1989).

Kinloch, A. J., C. C. Lau and J. G. Williams, "The Peeling of Flexible Laminates," International Journal of Fracture, 66, 45-70 (1994).

Li, S., J. Wang and M. D. Thouless, "The Effects of Shear on Delamination of Beam-Like Geometries," Journal of the Mechanics and Physics of Solids, 52, 193214 (2004).

Parmigiani, J. P and M. D. Thouless, "The Effects of Cohesive Strength and Toughness on Mixed-mode Delamination of Beam-Like Geometries," Engineering Fracture Mechanics, in press (2007).

Suo, Z. and J W Hutchinson, "Interface Crack Between Two Elastic Layers," International Journal of Fracture, 43, 1-18, (1990). 
Thouless, M. D. and H. M. Jensen, "Elastic Fracture Mechanics of the Peel-Test Geometry," Journal of Adhesion, 38, 185-197 (1992).

Tvergaard, V. and J. W. Hutchinson, "The Relation between Crack Growth Resistance and Fracture Process Parameters in Elastic-Plastic Solids," Journal of the Mechanics and Physics of Solids, 40, 1377-1397 (1992).

Tvergaard, V. and J. W. Hutchinson, "On the Toughness of Ductile Adhesive Joints," Journal of the Mechanics and Physics of Solids, 44, 789-800 (1996).

Wei, Y. and J. W. Hutchinson, "Interface Strength, Work of Adhesion and Plasticity in the Peel Test," International Journal of Fracture, 93, 315-333, (1998).

Wei, Y., "Modeling Nonlinear Peeling of Ductile Thin Films - Critical Assessment of Analytical Bending Models using FE Simulations," International Journal of Solids and Structures, 41, 5087-5104 (2004).

Yang, Q. D., M. D. Thouless and S. M. Ward, "Analysis of the Symmetrical 90Peel Test with Extensive Plastic Deformation," Journal of Adhesion, 72, 115-132 (2000).

Yang, Q. D., M. D. Thouless and S. M. Ward, "Numerical Simulations of Adhesively-Bonded Beams Failing with Extensive Plastic Deformation," Journal of the Mechanics and Physics of Solids, 47, 1337-1353 (1999).

Yang, Q. D. and M. D. Thouless, "Mixed-mode fracture analyses of plasticallydeforming adhesive joints," International Journal of Fracture, 110, 175-187 (2001).

Yu H. H. and J. W. Hutchinson, "Influence of Substrate Compliance on Buckling Delamination of Thin Films," International Journal of Fracture, 113, 39-55 (2002).

Williams, J. G., "Root rotation and plastic work effects in the peel test", Journal of Adhesion, 41, 225 -238 (1993). 


\section{Figure Captions}

Figure 1 A schematic illustration of the peel-test geometry. A film of thickness $h$, modulus $E$ and Poisson's ratio $v$ is bonded to a substrate of modulus $E_{s}$ and Poisson's ratio $v_{\mathrm{s}}$. A force $P$ is applied to the remote end of the film at an angle $\theta$ to the plane of the interface between the film and substrate.

Figure 2 Mode-I and mode-II traction-separation laws used for the cohesivezone model in this paper.

Figure 3 A beam on an infinite substrate subjected to (a) an axial load, $N$ (per unit width), (b) a bending moment, $M$ (per unit width), and (c) a transverse shear force, $V$ (per unit width) acting at the crack tip.

Figure 4 The relationship between the forces and moment acting at the crack tip, and the applied load and geometry of a peel test.

Figure 5 Plots of bending moment as a function of the peel strain for a peel angle of $90^{\circ}$ and modulus mismatches given by $\alpha=-0.8,0$ and 0.8 $(\beta=0)$. A comparison to the result that neglects the effects of shear is shown.

Figure 6 Plots of root rotation as a function of peel strain for a peel angle of $90^{\circ}$ and modulus mismatches given by $\alpha=-0.8,0$ and $0.8(\beta=0)$.

Figure 7 Plots of phase angle as a function of the peel angle for different peel strains.

Figure 8 Plots of yield strain against normalized adherend thickness showing the regime in which plasticity does not occur.

Figure 9 The results of cohesive-zone analyses showing how the peel force depends on the normalized film thickness for a $90^{\circ}$-peel test. The following dimensionless parameters were used: $\alpha=\beta=0 ; n=0.2$; $\theta=90^{\circ} ; \hat{\tau} / \sigma_{Y}=1 ; \sigma_{Y} / \bar{E}=0.00130 ;$ and $\Gamma_{\mathrm{II}} / \Gamma_{\mathrm{I}}=1$. The uncertainties 
indicated on this plot are associated with the uncertainties of the numerical analyses.

Figure 10 (a) Distribution of von Mises stresses in the film at the onset of crack propagation during a peel test with $\hat{\sigma} / \sigma_{Y}=3$ $\alpha=\beta=0, n=0.2, \theta=90^{\circ}, \hat{\boldsymbol{\tau}} / \sigma_{Y}=1, \sigma_{Y} / \bar{E}=0.00130, \Gamma_{\mathrm{II}} / \Gamma_{\mathrm{I}}=1$, and $\sigma_{Y} h / \Gamma_{I}=4.76$. The stresses in this plot have units of $\mathrm{MPa}$, and can be normalized by the yield stress of $100 \mathrm{MPa}$. (b) Maximum logarithmic shear strain distribution at the same point, showing the distortion of the mesh. The deformation is in true scale, with no magnification. 


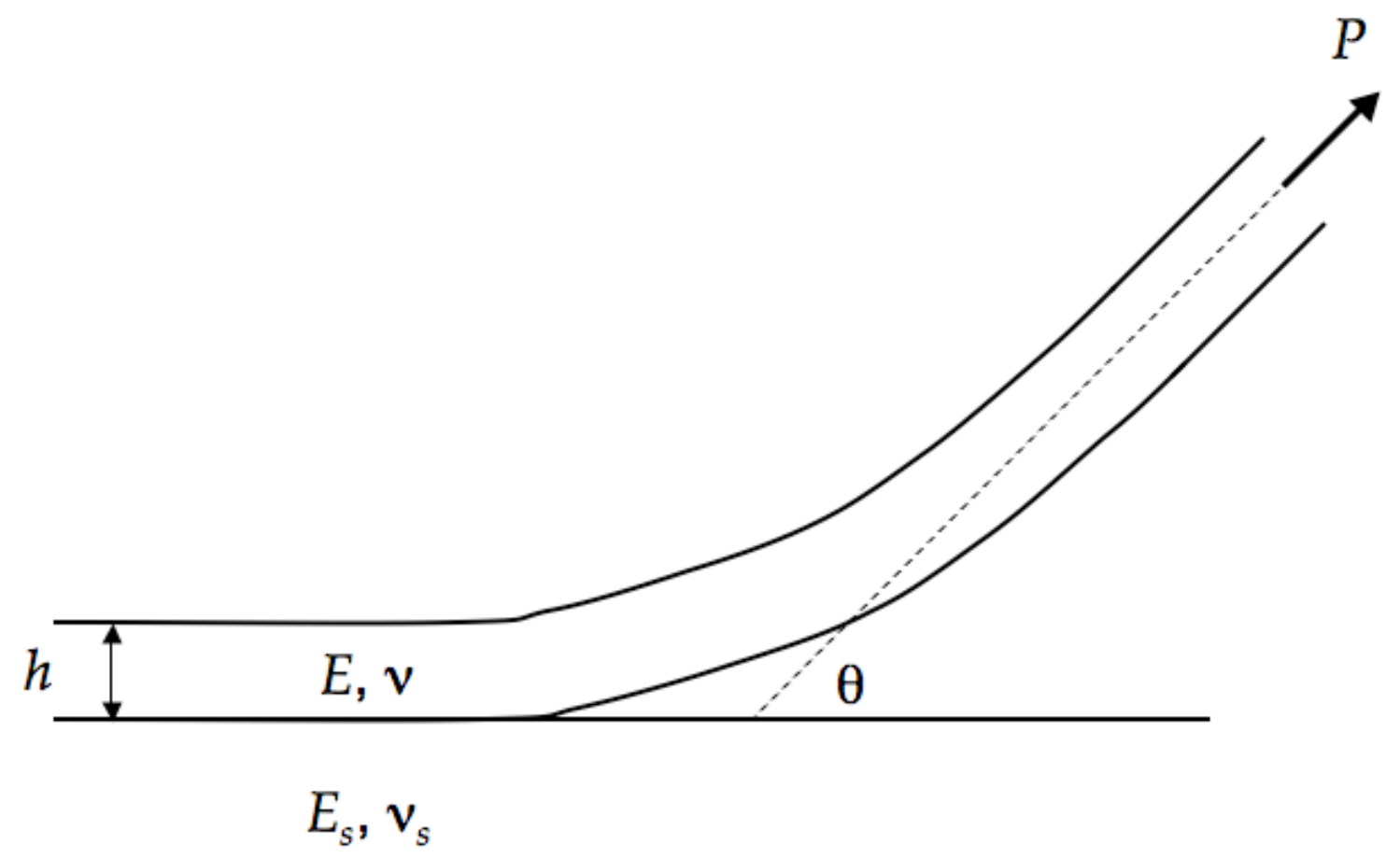

Figure 1 

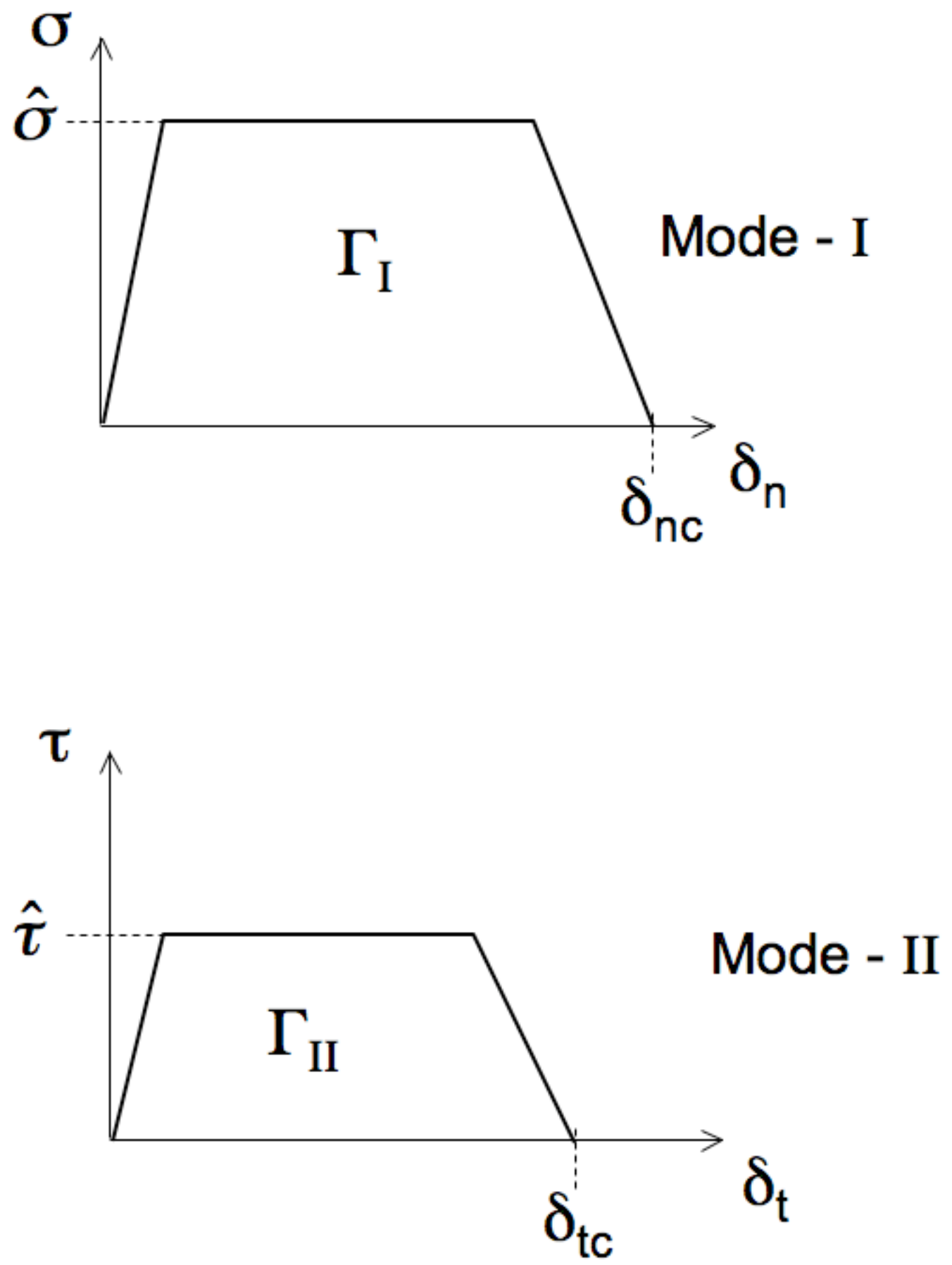

Figure 2 
a)

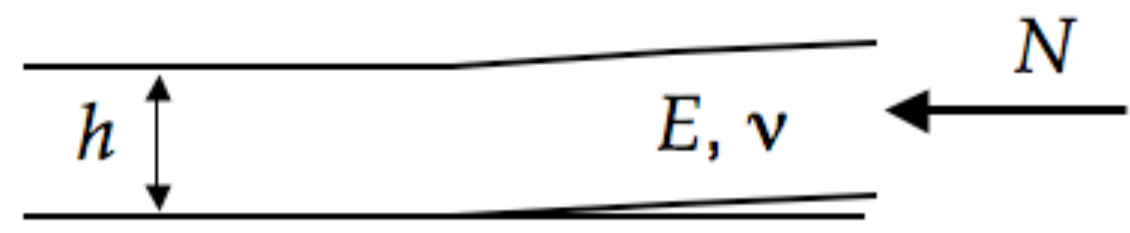

b)

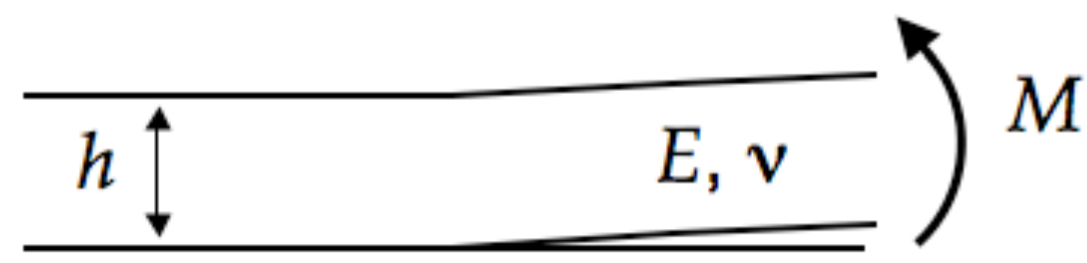

c)

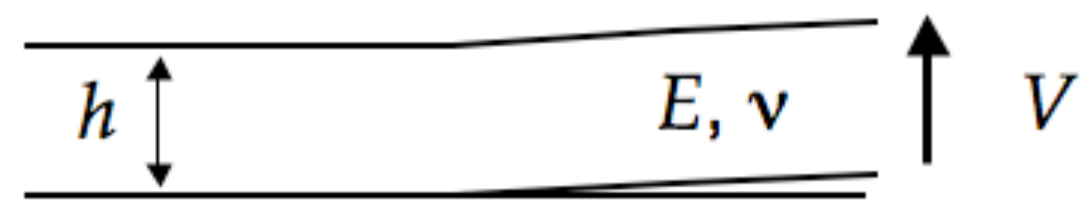

Figure 3 


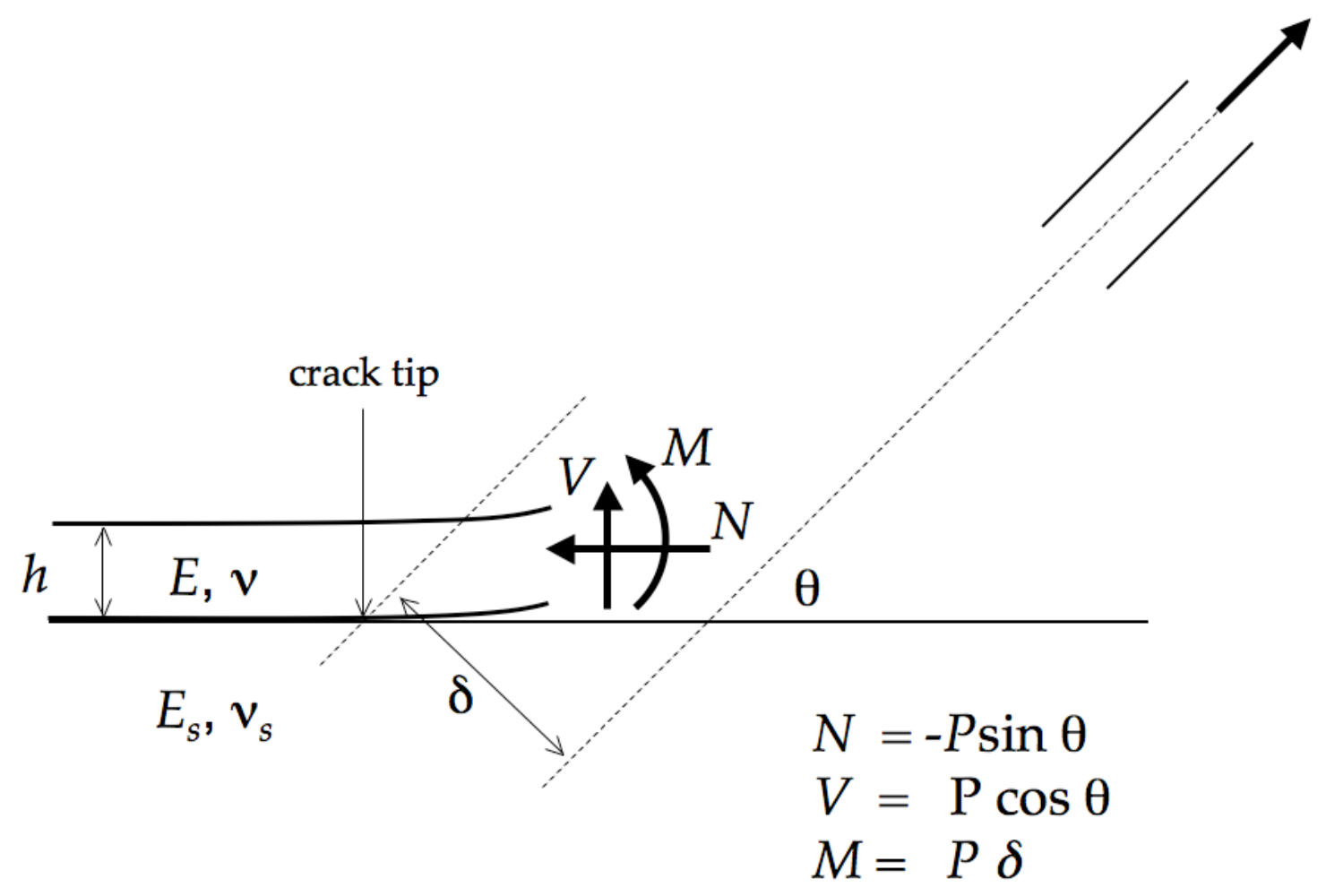

Figure 4 


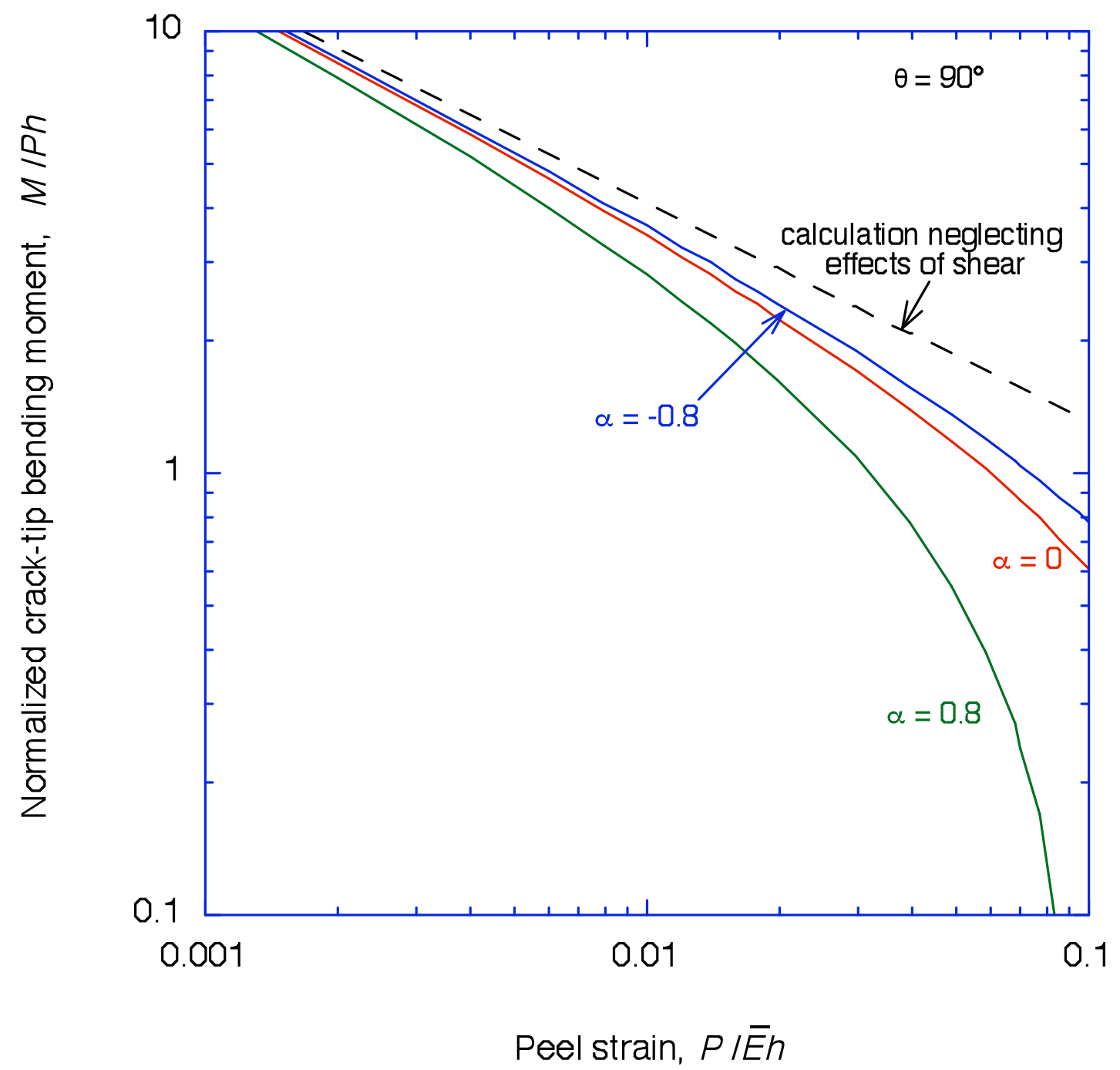

Figure 5 


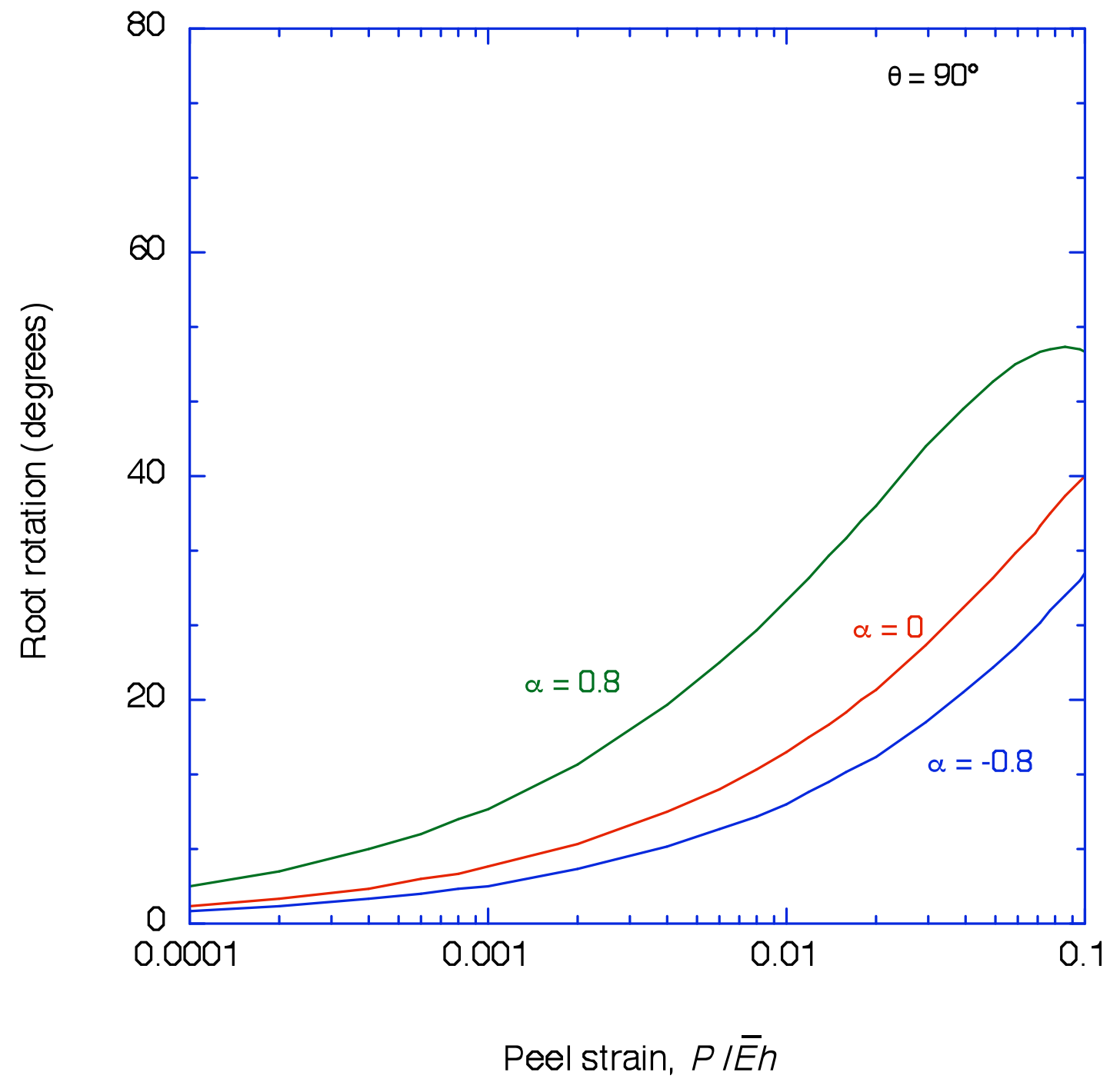

Figure 6 


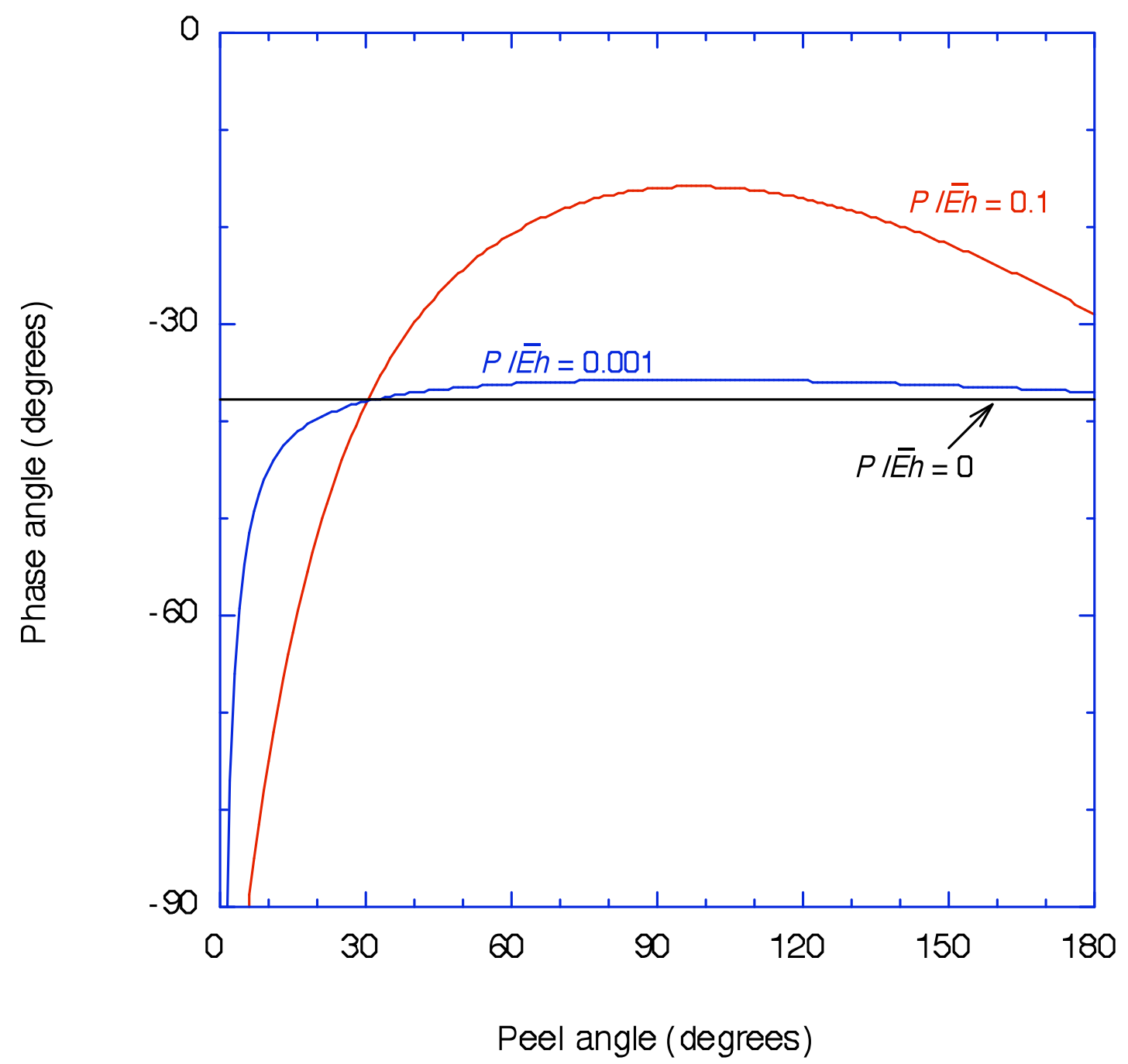

Figure 7 


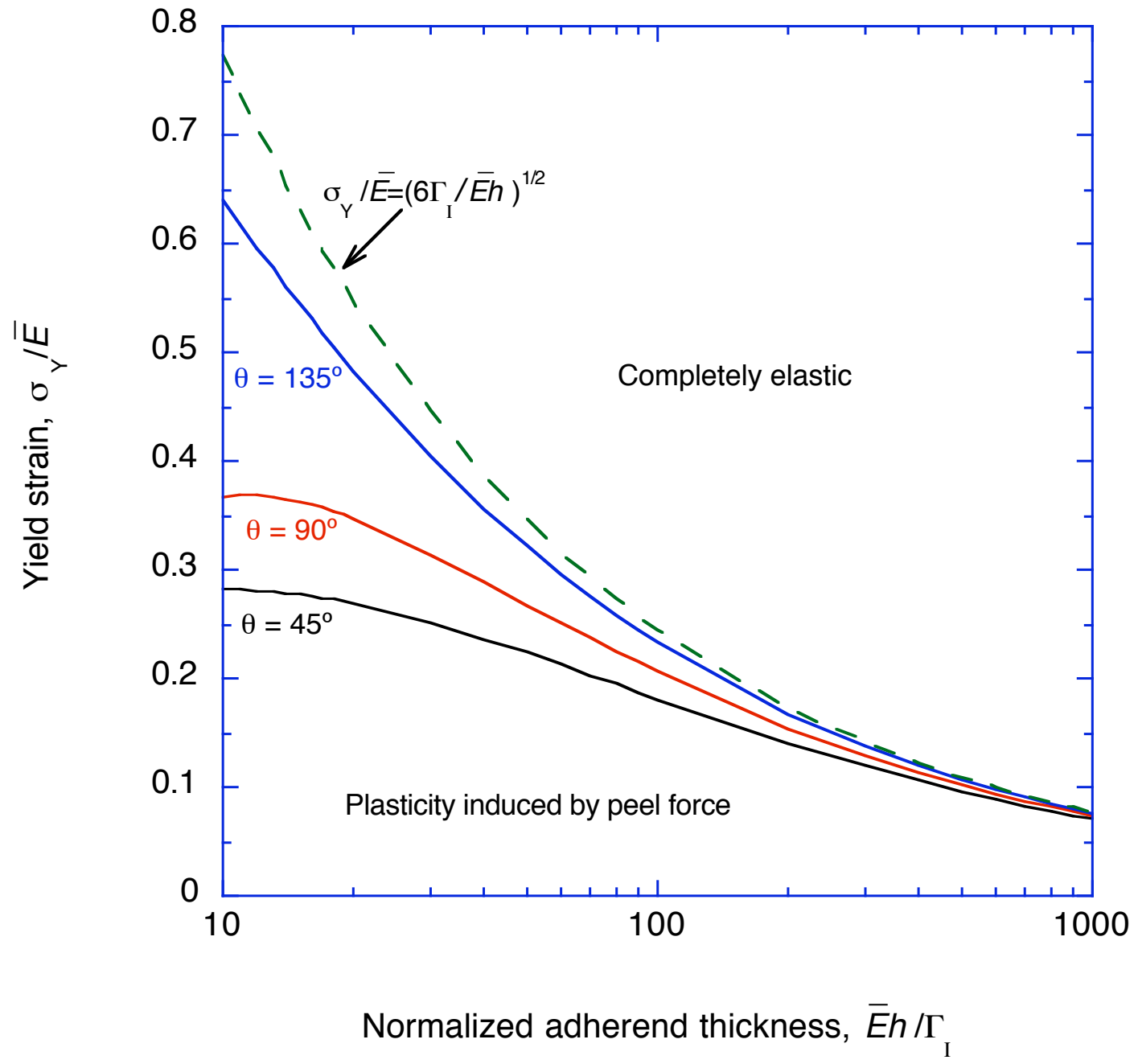

Figure 8 


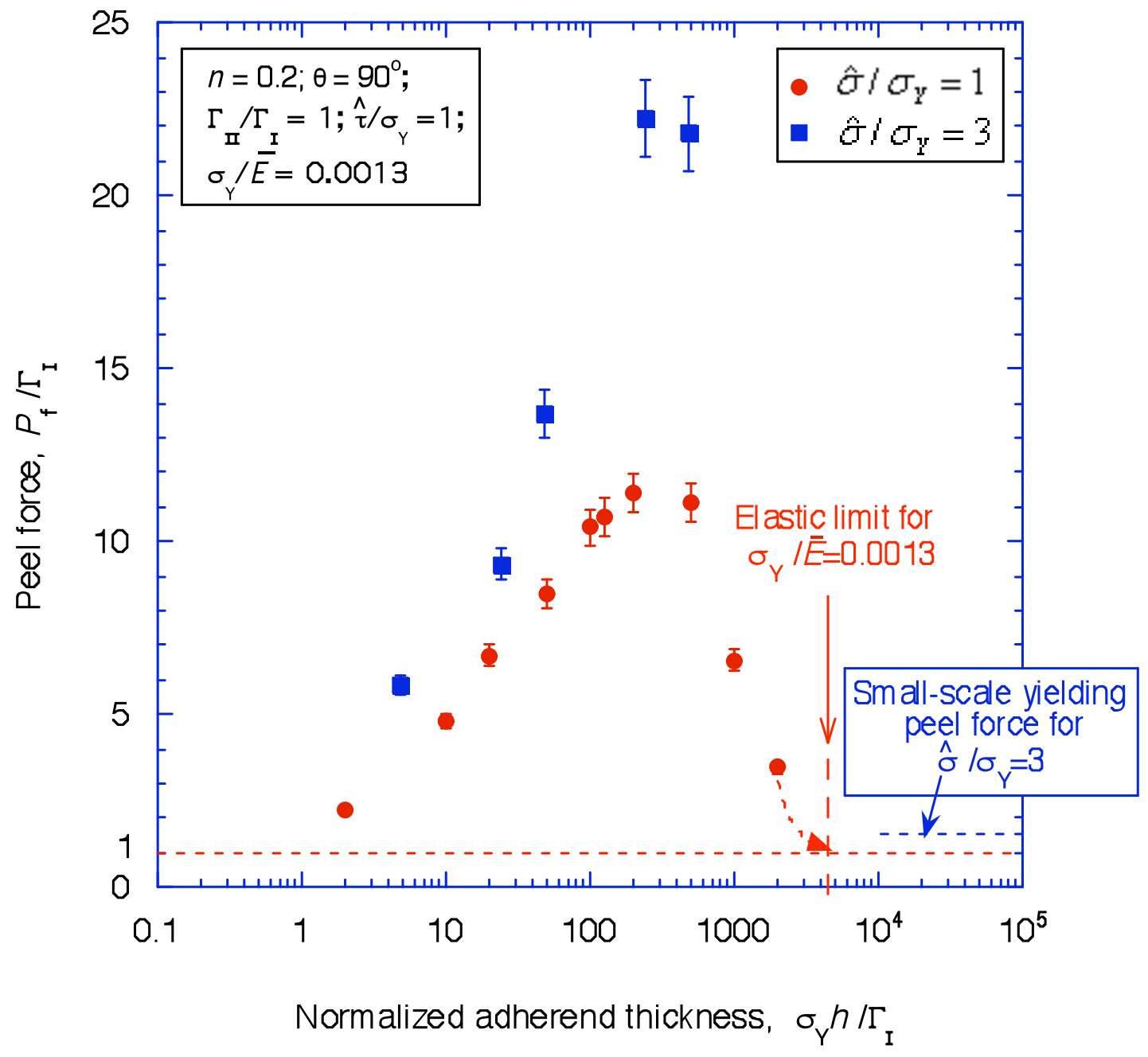

Figure 9 


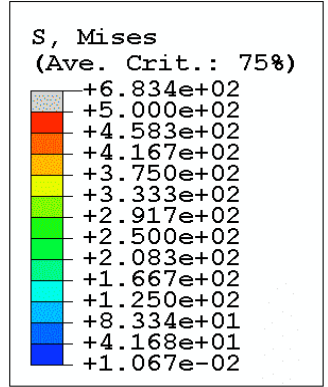

Initial crack-tip

(a)

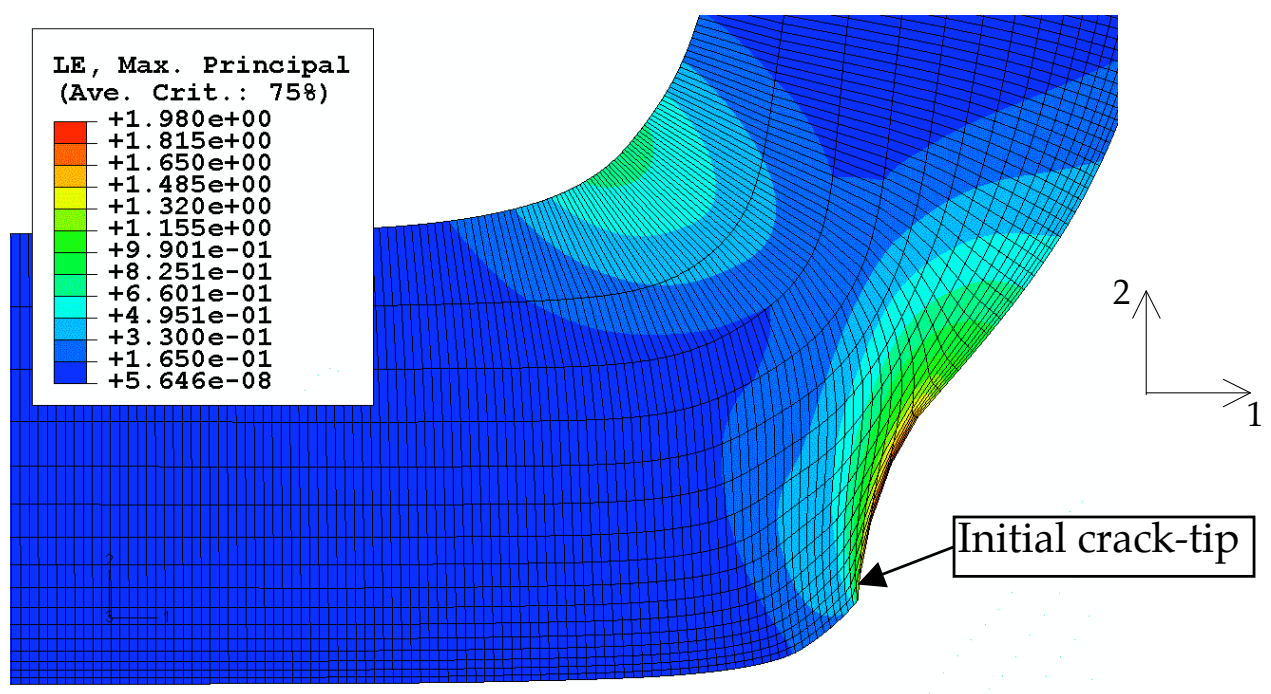

(b)

Figure 10 